\title{
Reportear bajo amenaza: violencia, profesionalización y modernización irregular del sistema mediático mexicano*
}

\section{Rubén Arnoldo González Macías ${ }^{1}$ \\ Elba Díaz-Cerveró ${ }^{2}$ Daniel Barredo-Ibáñez ${ }^{3}$}

Recibido: 23/08/2019

Aprobado por pares: 19/09/2019
Enviado a pares: 28/08/2019

Aceptado: 08/10/2019

DOI: 10.5294/pacla.2021.24.1.1

Para citar este artículo / to reference this article / para citar este artigo González, R. A., Díaz-Cerveró, E. y Barredo-Ibáñez, D. (2021). Reportear bajo amenaza: violencia, profesionalización y modernización irregular del sistema mediático mexicano. Palabra Clave, 23(4), e2411. https://doi.org/10.5294/pacla.2021.24.1.1

\section{Resumen}

Un sistema mediático considerado como moderno requiere, entre otros aspectos, el ejercicio de un periodismo libre y profesional. Sin embargo, en México, esto no ha sido del todo posible, porque la creciente violencia contra los periodistas se ha erigido como uno de los principales obstáculos para su trabajo. En ese sentido, a partir de 93 entrevistas semiestructuradas realizadas en 23 de los estados más violentos del país, el objetivo es describir las respuestas de los periodistas mexicanos hacia las constantes amenazas y agresiones que enfrentan. Los resultados indican que, en relación con la profesionalización del periodismo, la violencia contra los medios tiene

\footnotetext{
Este trabajo fue financiado por la Secretaría de Educación Pública de México, a través del programa Nuevo Profesor de Tiempo Completo. Clave: BUAP-PTC-521. Los autores agradecen al doctor Simon Cottle (Universidad de Cardiff, Reino Unido) por su valioso apoyo en el diseño y conducción del estudio. Asimismo, reconocen el trabajo de Luis García, asistente de investigación, quien realizó todas las entrevistas.

1 https://orcid.org/0000-0002-6758-5328. Benemérita Universidad Autónoma de Puebla, México. ruben.arnoldo@correo.buap.mx

2 https://orcid.org/0000-0002-0238-1498. Universidad Panamericana, México. eldiazc@up.edu.mx

3 https://orcid.org/0000-0002-2259-0756. Universidad del Rosario, Colombia. daniel.barredo@urosario.edu.co
} 
principalmente dos implicaciones. En primer lugar, los ataques han favorecido el desarrollo de mejores prácticas periodísticas, tales como apego a los datos y hechos, diversificación de fuentes de información, despliegue de coberturas colaborativas y creación de asociaciones de periodistas. En segundo lugar, en no pocos casos, la violencia ha inhibido el ejercicio de la prensa, fomentando la autocensura o la dependencia de los boletines oficiales de gobierno. La coexistencia de ambas reacciones pone de manifiesto el carácter irregular del sistema mediático mexicano y su proceso de modernización.

\section{Palabras clave (Fuente: tesauro de la Unesco)}

Periodismo; violencia; profesionalización; modernización; sistema mediático; múltiples periodismos. 


\section{Reporting under Threat:}

Violence, Professionalization,

and Erratic Modernization of

the Mexican Media System*

\section{Abstract}

A media system deemed modern requires, among other things, the practice of free professional journalism, which has not been entirely possible in Mexico because the growing violence against journalists has emerged as one of the principal obstacles to their job. The aim is to describe Mexican journalists' responses to the relentless threats and attacks they face based on 93 semi-structured interviews conducted in 23 of the country's most violent states. Concerning journalism professionalization, the results suggest that violence against the media has two implications mainly. First, the attacks have favored the development of better journalistic practices, such as adherence to data and facts, diversification of information sources, deployment of collaborative coverage, and creation of journalist associations. Second, in a significant number of cases, violence has inhibited press freedom, promoting self-censorship or dependence on official government newsletters. The coexistence of both reactions reveals the fickle nature of the Mexican media system and its modernization process.

\section{Keywords (Source: Unesco Thesaurus)}

Journalism; violence; professionalization; modernization; media system; multiple journalism.

\footnotetext{
* This research was funded by the Mexico Public Education Secretary's Office through the New Full-Time Professor program. Key: BUAP-PTC-521. The authors thank Dr. Simon Cottle (Cardiff University, UK) for his valuable support in the design and conduct of the study. They also acknowledge the work of Luis García, research assistant, who conducted all the interviews.
} 


\section{Fazer jornalismo sob ameaça: violência, profissionalização e modernização irregular do sistema midiático mexicano*}

\section{Resumo}

Um sistema midiático considerado moderno requer, entre outros aspectos, o exercício de um jornalismo livre e profissional. No entanto, no México, isso não tem sido totalmente possível, porque a crescente violência contra os jornalistas tem se tornado um dos principais obstáculos para seu trabalho. Nesse sentido, a partir de 93 entrevistas semiestruturadas realizadas em 23 dos estados mais violentos do país, tem-se o objetivo de descrever as respostas dos jornalistas mexicanos às constantes ameaças e agressões que enfrentam. Os resultados indicam que, com relação à profissionalização do jornalismo, a violência contra os meios de comunicação tem principalmente duas implicações. Em primeiro lugar, os ataques têm favorecido o desenvolvimento de melhores práticas jornalísticas, tais como o apego aos dados e fatos, a diversificação de fontes de informação, a implementação de coberturas colaborativas e a criação de associações de jornalistas. Em segundo lugar, em não poucos casos, a violência tem inibido o exercício da imprensa, fomentando a autocensura ou a dependência dos boletins oficiais de governo. A coexistência de ambas as reações revela o caráter irregular do sistema midiático mexicano e seu processo de modernização.

\section{Palavras-chave (Fonte: tesauro da Unesco)}

Jornalismo; violência; profissionalização; modernização; sistema de mídia; múltiplos jornalismos.

* Este trabalho foi financiado pela Secretaria de Educação Pública do México, por meio do programa Nuevo Profesor de Tiempo Completo. Chave: BUAP-PTC-521. Os autores agradecem ao doutor Simon Cottle (Universidade de Cardiff, Reino Unido) por seu valioso apoio na elaboração e condução do estudo. Igualmente, reconhecem o trabalho de Luis García, assistente de pesquisa, que realizou todas as entrevistas. 


\section{Introducción}

Ejercer el periodismo en México es una actividad de alto riesgo. Tan solo en la última década más de cien periodistas han sido asesinados, sin mencionar las innumerables amenazas y otros tipos de agresiones a las que constantemente se deben enfrentar (Article 19, 2019; Committee to Protect Journalists [CPJ], 2019). A lo anterior se suma una casi completa impunidad de la que gozan los agresores, porque menos de uno de cada diez ataques deriva en una condena, y muchos ni siquiera son investigados adecuadamente.

Para mantenerse a salvo ante esta situación, los periodistas mexicanos se enfrentan a un dilema al hacer su trabajo: profesionalizan su actividad periodística o se autocensuran. En ese sentido, el objetivo es describir ambas alternativas y sus implicaciones para la modernización del sistema mediático mexicano. Para lograrlo, esta investigación se sustenta en una base de datos compuesta por 93 entrevistas semiestructuradas con periodistas de los 23 estados más peligrosos del país.

El principal aporte estriba en explicar que la respuesta de los informadores mexicanos al riesgo constante de sufrir un ataque no siempre es el repliegue de su labor. Por el contrario, y a diferencia de gran parte de la literatura sobre este tema (Cottle et al., 2016; Holland y Ríos, 2015; Del Palacio, 2018), los hallazgos presentados también sugieren que, en lugar de resignarse, algunos medios y periodistas se esfuerzan por recuperar el control sobre su trabajo a través de la profesionalización. Esto apunta hacia un replanteamiento de su posición con respecto del problema: no solo son víctimas, sino también agentes activos en su profesión. Además, las discusiones sobre la profesionalización periodística se han enfocado principalmente en entornos no violentos (Deuze, 2005; Gans, 2004; Meijer, 2012), pero su ejercicio en condiciones de riesgo como el descrito aún no ha sido analizado en profundidad.

No obstante, como se discutirá, esta situación no es generalizada para todo el sistema mediático mexicano, en el que, a pesar de ciertos visos de un ejercicio periodístico más moderno, aún persisten inercias que ralenti- 
zan este cambio. Tal situación permite la existencia de prácticas periodísticas diferenciadas, que indican un proceso irregular de modernización de la prensa en México.

Para desarrollar las ideas expuestas, el contenido se organiza de la siguiente manera: en la primera sección, se presenta una revisión de la literatura enfocada en los conceptos de profesionalización periodística, modernización mediática irregular y violencia contra la prensa. Posteriormente, se describirá la metodología con la que se realizó el trabajo de campo, seguida del análisis de los resultados. Finalmente, se cierra con las conclusiones.

\section{Revisión de la literatura}

\section{Profesionalización periodística: definición y características}

En términos periodísticos, la profesionalización es un concepto difuso. Tanto los académicos como quienes ejercen la profesión de informar han tratado de definirla, pero no se ha alcanzado un acuerdo que satisfaga a todos. A pesar de ello, se puede delinear una discusión lo suficientemente robusta que permita dar sentido a los resultados de este estudio, que serán presentados más adelante.

Tomando como primer peldaño el ya clásico trabajo de Tuchman (1978), este término "connota el ejercicio de la autonomía, el derecho de los trabajadores a controlar su propio trabajo, frecuentemente en referencia a normas desarrolladas por grupos de profesionales externos a las organizaciones en las que los periodistas trabajan" (p. 65). ${ }^{4}$ En ese sentido, el primer elemento clave es la socialización, tanto al interior como al exterior de las redacciones. De ahí que "los reporteros aprenden lo que sus organizaciones esperan de ellos a través de la observación y experiencia [... Los nuevos periodistas rápidamente aprenden de sus compañeros más experimentados qué es lo que le gusta a su jefe" (Shoemaker y Reese, 1996, p. 92). Puesto que compartir e internalizar las rutinas particulares de cada medio es la manera más efectiva de aprender el oficio, la socialización es, entonces, importante para entender este término.

4 Las traducciones son nuestras. 
Otro concepto relevante asociado con la profesionalización periodística es el de la objetividad, el cual ha generado un debate interminable con respecto a su existencia y alcance. A pesar de que en la práctica no hay tal cosa llamada objetividad periodística, no pocos reporteros en todo el mundo aún se aferran a la creencia de que, si presentan la información de forma "distante, balanceada e impersonal" (Tuchman, 1972, p. 676), están siendo objetivos. No obstante, la realidad apunta a otra dirección, puesto que estos cánones tan valorados son el resultado de acuerdos internos, alcanzados por personas con sus propios intereses y guiados por sus propias ideologías, es decir, sus propias subjetividades. Sin embargo, para prevenir críticas tanto internas como externas, los periodistas están más que dispuestos a seguir los estándares que han aprendido en forma de rutinas, las cuales determinan sus actividades diarias: ofrecer más de una versión de la historia, presentar datos extra, citar directamente a sus fuentes, estructurar el texto en orden de importancia descendente y separar claramente los datos de las opiniones (Tuchman, 1972). Por tanto, la objetividad demanda cumplir con todos estos cánones, que se pueden resumir en dos requisitos principales: por un lado, el apego a los datos duros, lo que implica las nociones de verdad y relevancia; por el otro, la imparcialidad, que es la presentación de la información de una manera balanceada y libre de tendencias políticas (Deuze, 2005; Gans, 2004; Meijer, 2012).

Por su parte, Hallin y Mancini (2004) propusieron tres dimensiones para evaluar el nivel de profesionalización de los periodistas que integran un determinado sistema mediático: autonomía, normas profesionales y orientación al servicio público. La primera advierte que, dada su condición de empleados no de empleadores, los reporteros nunca tendrán un control completo de su trabajo. Sin embargo, sí pueden llegar a obtener un grado importante de autonomía cuando de forma individual o colegiada logran conciliar sus intereses con los de la organización para la que trabajan. El segundo aspecto sugiere la existencia de principios éticos en común en cada sistema mediático, tales como la clara separación entre publicidad e información, o la confidencialidad de ciertas fuentes, entre otros criterios periodísticos. Finalmente, la tercera dimensión representa el grado en el que los periodistas asumen el rol de servicio público. Esto significa 
responder al derecho de las audiencias a estar informadas sobre los asuntos de interés público.

En concreto, la profesionalización periodística representa la observancia de una serie de cánones compartidos y aceptados por periodistas con la preparación necesaria para desarrollarse en un sistema mediático. Dichos cánones son conocidos como rutinas, cuya finalidad es estructurar el trabajo de los involucrados en el proceso de producción de noticias (reporteros, fotógrafos, editores, conductores, entre otros). Algunos de estos estándares, por ejemplo, comprenden acciones como ofrecer más de una versión de los hechos, presentar datos suplementarios, verificación exhaustiva de la información, uso de citas directas de los entrevistados, en conjunto con la separación entre los hechos y las opiniones. Todos estos requerimientos se incluyen en el contenido de un mensaje impersonal, balanceado, informativo y exacto (Deuze, 2005; Gans, 2004; Meijer, 2012).

\section{Modernización irregular del sistema mediático mexicano}

Al igual que la noción de profesionalización, el concepto de modernización es problemático dado que su empleo difiere de un contexto a otro y, por ende, su significado preciso carece de consenso. Por ejemplo, esta palabra es ampliamente utilizada como sinónimo de evolución, desarrollo, globalización e, incluso, americanización. Además, a menudo es tomada como un concepto dicotómico que necesariamente implica su idea opuesta, la tradición. De modo que todo aquello que no es moderno deberá ser visto como tradicional o arcaico (Eisenstadt, 2000; Portes, 1973).

En la práctica, no hay solo una sino varias formas en que una nación puede volverse moderna, a pesar de que hay una tendencia a considerar como sinónimas las nociones de modernización y occidentalización, especialmente como procesos unidireccionales. En otras palabras, en lugar de una modernidad única, existen diferentes modernidades al interior de y entre los países (Eisenstadt, 2000). Esto significa que la modernización no es el resultado de la mera imposición o adopción de valores y estándares extranjeros, sino que implica la existencia de un contexto que favorezca o impida ese proceso. Por tanto, cada sociedad puede alcanzar este punto a través 
de su muy particular conjunto de condiciones exógenas y endógenas, tal y como se presenta dentro y fuera del mundo occidental.

Una vez señaladas las limitaciones conceptuales, importa ahora avanzar hacia una definición de este término. A pesar de la diversidad de aproximaciones, se puede considerar la modernización como un proceso de cambio social, asociado a nociones como de crecimiento económico basado en la industrialización, democratización del sistema político, organización burocrática de las instituciones, movilidad social a partir de la educación y existencia de una prensa liberal, entre otros aspectos. Más allá del cambio per se, esta idea también puede ser considerada como una transformación orientada hacia metas específicas, y liderada por élites políticas y económicas (Eisenstadt, 2000; Portes, 1973).

Por lo que respecta a la investigación en el área de periodismo, a fines del siglo XX e inicios del siglo XXI, diferentes autores han coincidido en que los sistemas mediáticos en todo el mundo se estaban alineando con los estándares del modelo liberal. Esto significa que en la operación de los medios se ha dado una convergencia de valores y prácticas, tales como comercialización, independencia política, apego a los datos y separación entre información y opinión, entre otros (Hallin y Mancini, 2004; Mancini y Swanson, 1996). La razón de lo anterior era que los sistemas políticos también se estaban moviendo en una dirección similar, caracterizada por el declive de la identidad partidista, el incremento del comercio internacional y la mediatización de la política, por mencionar solo algunos aspectos. Sin embargo, estudios más recientes han cuestionado este argumento, especialmente en los países en desarrollo, cuyos sistemas mediáticos, a pesar de una oleada general de democratización, no necesariamente se han modernizado y en los que conviven rasgos manifiestos de su pasado autoritario (Sparks, 2011). En otras palabras, en lugar de transitar directamente del autoritarismo al liberalismo, no pocos medios en el Sur global han adoptado formas híbridas de hacer periodismo, que presentan diferentes combinaciones de cambios y continuidades. Lo anterior también puede ser considerado como un reflejo del sistema político al que pertenecen, el cual formalmente puede ser considerado como democrático, aunque, en la práctica, está li- 
mitado por resabios autoritarios. De igual forma, esto último es evidente en el ámbito económico, en el que las corporaciones transnacionales coexisten junto al comercio informal y otras actividades económicas paralegales.

En ese sentido, y siguiendo la mencionada noción de múltiples modernidades, la práctica periodística también puede caracterizarse como heterogénea antes que homogénea. Por ende, se puede dar el caso de la convivencia de múltiples periodismos en un mismo sistema mediático. Más allá de su falta de homogeneidad, tanto en el interior de un país como entre varias naciones, los medios en su conjunto son diversos, no son estáticos, y tampoco pueden reducirse a las partes que los integran, porque están profundamente vinculados al contexto que los rodea (De Albuquerque, 2018; Waisbord, 2006).

Por tanto, entendida como un proceso continuo de cambio, la modernización de los medios es resultado de una combinación de fuerzas exógenas (principalmente relacionadas con la democratización del sistema político y la liberalización de la economía) y endógenas (propias de la profesión periodística), que, cuando sincronizan y acoplan su desarrollo, facilitan la transformación general del sistema. Empero, esta situación de convergencia armónica de ambos tipos de fuerzas es más la excepción que la regla, tal y como lo demuestra el caso mexicano, ya que, en el contexto de las nuevas democracias, es más probable que se dé una colisión entre ellas (González y Echeverría, 2018; Voltmer, 2012).

Como resultado de la interacción entre los factores que promueven la modernización (alternancia partidista, liberalización económica, privatización de medios estatales, etc.) y aquellos que la impiden (clientelismo, violencia, concentración de medios, etc.), surge una mezcla de cambios y continuidades que determinan la organización y operación de las empresas de comunicación. Sin embargo, en países extensos como México, estas condiciones no pueden generalizarse a lo largo y ancho del territorio nacional, puesto que cada región presenta una particular combinación de fuerzas que fomenta la transformación y, además, otras que la obstaculizan. En concreto, en lugar de un sistema mediático nacional homogéneo, existen 
varios subsistemas heterogéneos con su propio proceso de modernización (González y Echeverría, 2018).

El caso mexicano es un ejemplo apropiado de este argumento, dado que en cada una de sus regiones coexisten diferentes niveles de fuerzas exógenas y endógenas (asociadas a los sistemas político y mediático, respectivamente), que determinan el ritmo y los alcances de la transformación. A pesar de que las características del sistema político y mediático están presentes en todas partes del país, los rasgos específicos de cada zona impulsan el cambio o la continuidad. Por esta razón, los medios de la Ciudad de México, asumidos como nacionales, tienden a ser más liberales que sus pares en los estados. Aunque, cabe apuntar, todos ellos también comparten algunos aspectos autoritarios (González y Echeverría, 2018; Salazar, 2018).

Considerar como irregular el proceso de modernización periodística en México no implica ni transformación ni atraso definitivos. Por el contrario, el proceso apunta a una diversidad de prácticas en el ejercicio del periodismo, moldeadas por una interacción diferenciada entre fuerzas exógenas y endógenas. Dicho de otra forma, existen múltiples periodismos y no un solo patrón más o menos consolidado de ejecución. En estos términos, resultaría poco realista esperar una modernización homogénea del sistema mediático mexicano cuando el propio país se encuentra a medio camino, cualitativa y geográficamente, de su transición democrática, lo cual también es resultado de la coexistencia de aspectos tanto liberales o democráticos como autoritarios (González y Echeverría, 2018; Salazar, 2018).

\section{Violencia contra los periodistas}

México es uno de los países más peligrosos del mundo para ejercer el periodismo. Asílo indican consistentemente diversos reportes tanto nacionales como internacionales, en los que se concluye que la libertad de prensa es muy limitada en comparación con otras naciones democráticas (Article 19, 2019; CPJ, 2019). Además, la violencia contra los periodistas se ha transformado en un fenómeno cada vez más complejo, en el que intervienen diversos actores (crimen organizado, gobierno, cacicazgos locales, movimientos sociales, grupos de interés, etc.) que ejercen distintos tipos de 
agresiones, que van desde las amenazas y difamación hasta golpizas, secuestros y asesinatos (Article 19, 2019; Cottle et al., 2016; Del Palacio, 2018).

Dada su naturaleza multifacética, el análisis de los ataques contra los informadores es problemático, especialmente cuando se trata de medirlos. Existe un creciente número de reportes, publicados por distintos organismos oficiales y no gubernamentales, que ofrecen resultados igualmente diversos (Article 19, 2019; CPJ, 2019). A pesar de las inconsistencias metodológicas, todos coinciden en, al menos, tres aspectos. En primer lugar, el número de agresiones es considerablemente alto si se compara con cualquier otro Estado democrático. En segundo lugar, muchas de las víctimas cubren temas relacionados con el crimen organizado o corrupción entre la clase política. Sin embargo, también se han dado algunos casos en otras fuentes, como información general, deportes y hasta espectáculos. En tercer lugar, la gran mayoría de esos ataques quedan impunes, puesto que menos de uno de cada 10 casos se resuelve, mientras que muchos ni siquiera son investigados por las autoridades (Brambila, 2017; Del Palacio, 2018).

Además, existen otros factores que complejizan aún más el caso mexicano: dispersión geográfica, corrupción, complicidad y diversidad de agresores. En cuanto al primer aspecto, solía considerarse que la violencia contra los periodistas mexicanos estaba focalizada solo en ciertas regiones, especialmente en aquellas zonas que eran disputadas por dos o más carteles (Brambila, 2017; Holland y Ríos, 2015). Sin embargo, en los últimos años, diversos asesinatos $y$ agresiones han tenido lugar en puntos que otrora eran considerados como seguros, tales como Ciudad de México, Baja California Sur, Quintana Roo o Chiapas. Por ende, han dejado de existir las ciudades santuario, porque el riesgo se ha expandido por todo el territorio nacional y, con ello, se ha generalizado la sensación de vulnerabilidad e indefensión entre los reporteros (González-Macías y Reyna-García, 2019).

Por lo que respecta a la corrupción y complicidad, durante no pocos años se han tejido redes de colaboración entre miembros del crimen organizado y ciertas autoridades gubernamentales, lo que ha derivado en que el 
periodismo mexicano se desarrolle en un estado endémico de indefensión. En otras palabras, el intercambio de favores entre estos actores con poder es el punto neurálgico del silenciamiento de los medios. Es decir, amedrentar a la prensa mediante amenazas, agresiones o falta de castigo a los culpables forma parte del acuerdo tácito de protección mutua (Article 19, 2019; CPJ, 2019; Del Palacio 2018; Hughes y Márquez, 2017; Relly y González, 2017). Finalmente, ante la casi absoluta impunidad, no solo los delincuentes y servidores públicos atacan a los periodistas. Por el contrario, cada vez se suman más agentes a la lista de agresores: Fuerzas Armadas, manifestantes, sindicatos, empresarios, entre otros (Article 19, 2019; CPJ, 2019).

\section{Metodología}

Este estudio es de corte cualitativo y se organiza a partir de una serie de entrevistas semiestructuradas con informadores mexicanos, realizadas entre febrero de 2017 y agosto de 2018. La muestra comprendida por 93 informantes, 62 hombres y 31 mujeres, fue seleccionada a partir de dos criterios. En primer lugar, debían ser periodistas en activo, empleados de alguna redacción ofreelancers. En segundo lugar, se requería que, en el momento de la entrevista, cubrieran, o hayan cubierto consistentemente hard news, especialmente temas de delincuencia organizada o corrupción. No fue criterio de exclusión su rol (p. ej., reportero, editor, fotógrafo, camarógrafo, conductor), ni el tipo de medio para el que trabajaran (periódico, revista, radio, televisión o portal de noticias).

La selección de entrevistados siguió la lógica del muestreo no probabilístico por bola de nieve, puesto que fueron los mismos periodistas quienes iban recomendado a otros colegas a participar en el estudio. Dentro de la conformación de la muestra final, se condujeron entrevistas con reporteros locales y corresponsales estatales de medios nacionales (ubicados en la Ciudad de México), tales como Proceso, La Jornada, Reforma, El Universal, Televisa, TV Azteca e Imagen Televisión. Asimismo, participaron periodistas de reconocidos medios locales y regionales, como El Informador, El Siglo, A.M., El Diario de Juárez, Zeta y Río Doce, por nombrar algunos. También se entrevistó a informadores de portales independientes de noticias locales, además de personal de agencias de noticias nacionales e internacionales (p. ej., Notimex, EFE y AFP). 
El cuestionario base aplicado estuvo enfocado en tres grandes temas. En el primero, se abordó el contexto general de la violencia en México. Aquí se preguntó, entre otras cosas, la razón por la que se ataca a la prensa, quiénes son los principales agresores y qué mecanismos de protección tienen los periodistas. En el segundo bloque, la atención se centró en el impacto individual, organizacional y social de la violencia contra los informadores. Finalmente, en el último apartado, se indagó el uso de la tecnología para proteger la seguridad personal y digital de los entrevistados. Cabe señalar que, debido a la vastedad de la información obtenida durante el trabajo de campo, se reportan principalmente los hallazgos referentes al segundo bloque de preguntas.

La decisión de optar por una metodología cualitativa se fundamenta en la necesidad de aproximarse al fenómeno desde el punto de vista de quienes se enfrentan cotidianamente al riesgo de ser agredidos por su trabajo. En ese sentido, se buscaba que los informantes se expresaran libremente sobre los temas abordados, para lo cual la entrevista semiestructurada resultó fundamental. En otras palabras, más que obligar a los sujetos a ceñirse a respuestas fijas y predeterminadas, como sería en una encuesta, aquí se les permitió que compartieran sus opiniones y vivencias en sus propios términos.

Por tanto, más que generalizaciones aplicables a grandes grupos, fin último del enfoque cuantitativo, lo que se planteó al inicio fue ahondar en las prácticas y percepciones de los entrevistados. Para lograrlo, se ponderó la profundidad y riqueza de las respuestas sobre el tamaño de la muestra. No obstante, como se señala más adelante, se obtuvo información de casi tres cuartas partes del territorio nacional, de tal suerte que la evidencia empírica recabada ofrece una mirada lo suficientemente amplia del fenómeno en México.

Precisamente, para que la investigación tuviera un alcance nacional, las entrevistas se distribuyeron geográficamente siguiendo, por un lado, la zonificación del Consejo Nacional de Seguridad Pública (CNSP) y, por otro, los reportes presentados por Article 19 en 2016 y 2017 (Article 19, 2019), en los que se señala el número de agresiones contra periodistas y el estado 
donde tuvieron lugar. En ese sentido, la distribución de la muestra se enfocó en las 23 entidades más peligrosas reportadas por esta organización no gubernamental (ONG), y divididas en las regiones propuestas por el antes mencionado programa del Gobierno Federal (tabla 1).

\section{Tabla 1. Distribución geográfica de la muestra}

\begin{tabular}{|c|l|}
\hline Zona & \multicolumn{1}{|c|}{ Muestra por estado seleccionado } \\
\hline Noroeste & Baja California: 9; Chihuahua: 6; Sinaloa: 6; Sonora: 5 \\
\hline Noreste & $\begin{array}{l}\text { Coahuila: 3; Nuevo León: 4; San Luis Potosí: 4; } \\
\text { Tamaulipas: 4 }\end{array}$ \\
\hline Occidente & Aguascalientes: 1; Guanajuato: 2; Jalisco: 6; Michoacán: 7; Zacatecas: 3 \\
\hline Centro & Ciudad de México: 4; estado de México: 2; Guerrero: 2; Morelos: 1; Puebla: 8 \\
\hline Sureste & Chiapas: 3; Oaxaca: 3; Quintana Roo: 4; Tabasco: 1; Veracruz: 5 \\
\hline
\end{tabular}

Fuente: elaboración propia.

Finalmente, debido a las consideraciones éticas con las que se realizó esta investigación, la identidad de los informantes se mantendrá en el anonimato. Es decir, puesto que el carácter sensible de las respuestas podría comprometer a los entrevistados, se determinó hacer referencia a ellos mediante la letra E (Entrevistado) y un número (p. ej., E12). Para contextualizar y dimensionar sus respuestas, solo se indicará si el entrevistado es hombre o mujer, su rol en el medio y la región donde labora.

\section{Resultados}

Como se mencionó, la violencia contra los periodistas es un fenómeno complejo y, por tanto, su impacto presenta varios ángulos. En ese sentido, las repercusiones derivadas de los ataques no solo afectan a las víctimas de forma particular, sino que también alcanzan a los medios para los que trabajan. Es decir, en términos de profesionalización, el entorno de riesgo permanente impacta tanto en el ámbito individual como organizacional. La razón es que, además de las víctimas directas, la operación dentro de las redacciones también se ve afectada cuando alguno de sus miembros es agredido. No obstante, mientras que en ciertos casos situaciones como estas fomen- 
tan la profesionalización del gremio, en otros la inhiben. Por ende, la presentación de los hallazgos se organizará a partir de ambas tendencias y en los niveles antes indicados.

\section{Los ataques promueven la profesionalización}

Para realizar sus actividades informativas de una manera más profesional, algunos periodistas mexicanos a nivel individual han implementado una serie de cambios en sus rutinas de trabajo, entre los que destacan los siguientes: evitar hacer entrevistas por vía telefónica y procurar que sean siempre cara a cara, ponderar los datos y hechos por encima de los dichos, mejorar la selección de las fuentes según la calidad de la información que puedan ofrecer, contrastar y verificar los datos a través de diferentes mecanismos, contar con una adecuada calendarización de temas, almacenar información sensible en diferentes dispositivos digitales y físicos, pero no compartirla por correo electrónico o en la nube.

En ese sentido, E38, reportero de un periódico local en la zona occidente, explicó que "como periodista debes ser muy cuidadoso. Debes ser imparcial todo el tiempo y evitar ser muy cercano a las fuentes. Esto es uno de nuestros errores más frecuentes”. Por esa razón, E58, reportera de un portal de noticia locales en la zona sureste, sugirió que los periodistas "debemos ser muy cuidadosos con el trabajo, muy respetuosos, porque, si decides indagar algo, debes estar atento a las posibles consecuencias. Debes planear con antelación para protegerte”.

Por lo que respecta al nivel organizacional, el impacto de la violencia se ha hecho patente en una serie de cambios en las redacciones. En otros términos, las amenazas y agresiones en contra de los periodistas han alterado el proceso de producción de noticias en los medios para los que trabajan. Por tanto, varios entrevistados compartieron diversas medidas que sus organizaciones han desarrollado para mejorar su trabajo. Dentro de las más recurrentes, están las siguientes: impulsar el periodismo de investigación o, por lo menos, el reporteo de datos no de declaraciones, publicar solo trabajos sólidos basados en los hechos, planear con antelación la cobertura de temas potencialmente riesgosos o, como lo planteó E64, director de una radiodifusora en la región noroeste, "dentro de la redacción hemos aprendi- 
do a 'estirar la liga'. Después de cada investigación dura, nos comportamos como 'buenos muchachos' y hablamos de las tortugas y mariposas. Cosas sin la mayor importancia, ya sabes. Después de un rato, cuando las cosas ya se calmaron, empezamos a investigar de nuevo. Esa es la manera en la que manejamos las cosas. Es la única forma de hacer periodismo por aqui".

Otra de las respuestas más constantes se refiere a la cobertura grupal de hechos que pudieran resultar peligrosos. Es decir, los reporteros y fotógrafos que se enfocan en este tipo de historias coordinan sus esfuerzos para hacer trabajo colaborativo, lo cual puede tomar dos direcciones. Por un lado, diversos medios pueden organizarse para investigar un mismo tema y presentarlo como resultado colectivo. En este caso, nadie tiene la exclusividad de la información, porque todos participaron en su producción. Por otro, quienes cubren noticias policiacas suelen moverse como grupo, y así minimizar los riesgos individuales: "La violencia nos ha forzado a juntarnos. Nos hemos dado cuenta de que somos más vulnerables cuando andamos solos. Ahora todos nos cuidamos y trabajamos juntos" (E45, reportera de un periódico local en la zona noroeste). En ese sentido, E25, mujer, periodista de investigación freelance en la zona centro, indicó: "Cuando arreció la violencia, nos tomó a todos desprevenidos, porque no estábamos ni preparados ni organizados. Ahora estamos formando alianzas entre nosotros que nos han ayudado a estar mejor preparados ante esta situación. Hemos aprendido a ser más estratégicos, pero somos conscientes de que no podremos ganar mientras no colaboremos entre nosotros".

Otro impacto a nivel organizacional que han generado los ataques contra la prensa es la creación y consolidación de diversas asociaciones de periodistas. Estos grupos formales ${ }^{5}$ tienen dos objetivos principales: primero, a través de capacitación constante, promueven la profesionalización y seguridad del gremio; y segundo, cuando uno de sus compañeros es víctima de alguna agresión, le ofrecen asesoría legal y acompañamiento durante el proceso de denuncia ante las autoridades. Al respecto, se pronunció E56, hombre, corresponsal en la zona sureste de una revista nacional: "Como periodistas, debemos estar siempre unidos, porque la fragmentación es nues-

5 Algunas de estas asociaciones son Periodistas de a Pie, Red de Periodistas de Juárez, Voz Alterna, Prensa Oaxaca, entre otras. 
tra principal debilidad. Estoy seguro de que nadie es inmune a cualquier ataque, pero se pueden prevenir algunas agresiones con la capacitación adecuada. Aquí es donde las asociaciones de periodistas son importantes, porque están tratando de ayudarnos".

Asimismo, existen otras agrupaciones que también promueven iniciativas de ley para la protección de los periodistas, especialmente en regiones de alto riesgo. Un caso interesante se encuentra en la zona del sureste, donde una de estas asociaciones organiza reuniones frecuentes con diferentes grupos de interés, como los sindicatos de maestros y taxistas, para sensibilizarlos sobre la labor de los reporteros y fotógrafos. La finalidad es que no los agredan mientras cubren sus manifestaciones, porque solo están haciendo su trabajo y, además, dan visibilidad a las demandas de dichos grupos. Aunado a lo anterior, en al menos tres regiones (noroeste, centro y sureste) algunas de estas asociaciones de informadores han creado portales online independientes, en los que pueden publicar aquellos trabajos que han sido vetados de los medios tradicionales.

Además de estas asociaciones formales, existe una multitud de grupos informales a nivel local, en los que reporteros, fotógrafos o camarógrafos de diferentes medios se cuidan entre sí. Por ejemplo, a través de WhatsApp están constantemente monitoreando sus propios movimientos. Esto ha resultado particularmente útil durante la cobertura de sucesos potencialmente peligrosos, como la información policiaca.

Ya sean formales o informales, estos grupos han demostrado ser útiles para la consolidación del gremio periodístico. En ese sentido, se puede señalar que su creación y consolidación es un impacto benéfico de las agresiones contra la prensa mexicana, tal como lo señaló E32, fotógrafo en la zona centro de una revista nacional: "El nacimiento de estos grupos de periodistas es un efecto positivo del contexto general de violencia”.

\section{Los ataques inhiben la profesionalización}

No todos los informadores y medios mexicanos han reaccionado de la misma manera, a pesar de los visos de profesionalización y unión. Por el contrario, la mayoría de los entrevistados estuvieron de acuerdo en que el impacto 
principal de la violencia en su trabajo es la autocensura. Es decir, como resultado de alguna amenaza o ataque, los periodistas se ven obligados a eliminar cierta información de sus notas o, peor aún, a no difundir algo que pueda ponerlos en una situación de riesgo. Una muestra de esta encrucijada queda expuesta en la siguiente respuesta: "El primer impacto de la violencia es la autocensura. Considero que este es el peor de los efectos, porque no es lo mismo que alguien trate de impedir que difundas algo. En ese caso, tú acabas por publicarlo de una manera u otra. No obstante, cuando eres tú quien censura tu propio trabajo, no hay poder humano que te obligue a decir lo que no quieres decir" (E11, hombre, periodista de investigación de un periódico en la zona centro).

Tal y como sucede a nivel individual, diversos medios también han implementado la censura organizacional como resultado de las constantes agresiones y amenazas. Dicho de otro modo, desde las mismas redacciones se ha tomado la decisión de no cubrir temas sensibles, como las actividades del crimen organizado. La razón de ello es que este tipo de noticias son particularmente peligrosas, porque los periodistas han quedado atrapados y vulnerables en medio de luchas entre diferentes actores (carteles, Fuerzas Armadas, autoridades de gobierno, entre otros). Así se percibe en las palabras de E57, director de un portal de noticias en la zona noroeste: "Al principio, no teníamos ninguna medida de protección o protocolo de seguridad con relación a la cobertura de temas delicados. Cuando desapareció Alfredo Jiménez, ${ }^{6}$ los medios se vieron obligados a autocensurarse, especialmente en lo que respecta a notas del crimen organizado. Nos dimos cuenta de que aquí no podíamos seguir cubriendo información sobre narcotráfico, de lo contrario, enfrentamos el riesgo de ser secuestrados. En este punto, hay una censura en los medios impuesta por el miedo". Sin embargo, las actividades del crimen organizado no son la única fuente potencialmente riesgosa. Por el contrario, los participantes de este estudio también señalaron que la cobertura de casos de corrupción de servidores públicos, ya sean ellos solos o en colusión con otros actores, representa una labor peligrosa para los pe-

6 José Alfredo Jiménez Mota fue un joven periodista de un estado en la zona noroeste, quien desapareció en abril de 2005. La información con la que se cuenta es que fue secuestrado y se considera muerto, aunque sus restos siguen sin ser encontrados. Se presume que la causa del ataque fue su cobertura sobre las actividades del crimen organizado en su región. 
riodistas mexicanos. E52, hombre, corresponsal en la zona noreste de una televisora nacional, resume el sentir de sus colegas: "Vivimos en un estado de riesgo permanente. Podemos ser silenciados y obligados a ocultar información. Como periodistas estamos indefensos ante el poder económico y político de muchos personajes. Esta es una lucha injusta, porque realmente quisiéramos decir la verdad siempre, pero nuestras limitaciones nos sobrepasan. Frecuentemente tu jefe simplemente te dice 'esta nota no pasa'. ¿Por qué? Pues porque hay muchos intereses en juego. Los dueños de los medios son empresarios, hombres de negocios”.

En atención a estas condiciones, el resultado de la censura organizacional es la práctica de un periodismo menos profesional. En otras palabras, el riesgo potencial de una respuesta violenta por parte de algún involucrado en una noticia inhibe la investigación periodística sobre ciertos temas sensibles, tales como las actividades de grupos criminales o los casos de corrupción de servidores públicos. No obstante, de acuerdo con los entrevistados, cuando una información no puede ser ignorada, se reduce su visibilidad en la agenda mediática, esto es, nunca se publica en la portada, ni aparece en los primeros segmentos de los noticieros. Además, la nota solo se basa en la versión oficial de las instituciones gubernamentales, lo que fomenta la dependencia de los boletines de prensa en detrimento del reporteo cotidiano.

\section{Conclusiones}

En este artículo se resaltó la idea de que la violencia contra la prensa es un fenómeno polifacético, por lo que su impacto no siempre es homogéneo ni inmediato. Por ende, el caso mexicano demuestra que las constantes agresiones y amenazas tienen una doble implicación con respecto a la profesionalización del periodismo: mientras que en ciertos casos los ataques han promovido mejores prácticas de reporteo, en otros, por el contrario, las han obstaculizado. Esta situación refuerza el concepto de periodismos múltiples que coexisten simultáneamente en un sistema mediático.

En cuanto al poder inhibidor de la violencia sobre el ejercicio profesional del periodismo, importa señalar que es uno de los aspectos que más atención académica ha recibido en los últimos años (Cottle et al., 2016; Del 
Palacio, 2018; Hughes y Márquez, 2017). Entre los aspectos más analizados, se encuentran el declive del periodismo de investigación y la excesiva dependencia en los boletines de prensa oficiales, por mencionar solo un par. En ese sentido, este trabajo reforzó tales argumentos y, además, agregó a la discusión otros temas menos abordados, tales como la censura individualy organizacional. No obstante, también hay otro ángulo que necesita ser mejor documentado, y que justo representa la contribución de este artículo al estudio de la violencia contra la prensa. De acuerdo con la evidencia empírica presentada, en algunos casos, el estado de riesgo constante ha promovido mejores prácticas de reporteo y una incipiente unión del gremio. Con respecto a lo primero, emerge una tendencia hacia la investigación periodística $\mathrm{o}$, al menos, la búsqueda de datos y no meras declaraciones institucionales, y la diversificación de fuentes. Por otro lado, el segundo aspecto tiene que ver con la creación y consolidación de asociaciones formales de periodistas y la existencia de grupos informales de quienes cubren las mismas fuentes. Ambas agrupaciones promueven la profesionalización, la seguridad y el trabajo colaborativo.

Este último punto es particularmente digno de destacar, ya que se ha vuelto un lugar común asumir que los informadores mexicanos son un gremio marcadamente fragmentado. Sin embargo, como una respuesta a los incesantes ataques, se percibe un movimiento hacia una práctica más colectiva y menos individualizada o competitiva. Los resultados sugieren que, en otras circunstancias, esta sinergia habría sido más difícil de lograr. Aunque esta dista aún de ser la regla, se consignaron varios ejemplos a lo largo del país.

Aunado a lo anterior, la profesionalización en ciernes aquí analizada invita a una revisión y actualización de aquellos argumentos que tienden a señalar solo las limitaciones históricas del periodismo en México. Con unas pocas excepciones, se ha asumido que la prensa de calidad en este país es más un anhelo que una realidad, tal y como se percibe en buena parte de la revisión de la literatura (González-Macías, 2018; Reyna, 2014; Salazar, 2017). Sin embargo, los hallazgos presentados apuntan a una incipiente profesionalización de la práctica periodística, que también convive con un periodismo más bien pasivo y capturado. 
En concreto, como resultado de la violencia endémica, la coexistencia de ambas lógicas le da sentido al concepto de periodismos múltiples. Es decir, en lugar de un desempeño general y uniforme de todo el sistema mediático, hay una diversidad de aproximaciones al proceso de producción de noticias. Siguiendo los parámetros propuestos por Hallin y Mancini (2004) para evaluar la profesionalización periodística, los resultados señalan que los periodistas mexicanos tienen niveles heterogéneos de autonomía, normas profesionales y orientación hacia el servicio público. Esta situación no solo se da en comparación con otros países, lo cual sería evidente y esperado, sino también en el interior del propio sistema mediático. Esto indica que la variedad de condiciones políticas, económicas y de seguridad en cada una de las regiones del territorio nacional genera una igualmente variada práctica periodística. A su vez, esto último consolida la idea de la modernización irregular del sistema mediático mexicano, cuya operación está determinada por la práctica de algunos cánones liberales, pero enmarcada en un entorno principalmente autoritario y clientelar.

En ese sentido, el concepto de modernización irregular es pertinente para explicar la existencia de las diferentes lógicas con las que se realiza el ejercicio periodístico en México. El caso de la violencia contra los medios y su personal ilustra la diversidad de respuestas posibles que este fenómeno puede generar. Es decir, se ha asumido en trabajos anteriores (Del Palacio, 2018; González-Macías, 2018; Holland y Ríos, 2015; Hughes y Márquez, 2017; Relly y González, 2014) que, por ejemplo, los ataques a la prensa derivados de la cooptación de las instituciones gubernamentales por las organizaciones criminales, o la difuminación del monopolio estatal del uso de la fuerza, han constituido uno de los factores que más han obstaculizado el desarrollo del periodismo profesional. Sin embargo, no se había considerado la posibilidad de que tal situación pudiera generar un efecto diferente. Es decir, se ha dado por sentado que las agresiones obligan a los informadores a replegarse y no reportar más allá de lo que los grupos en el poder les indiquen. No obstante, la evidencia empírica presentada sugiere otra lectura: más que meras víctimas, algunos periodistas han sabido explotar su poder de agencia a su favor. En otras palabras, en lugar de resignarse a la autocensura o la dependencia del boletín oficial de gobierno, varios participantes 
de este estudio han adoptado un rol proactivo y, como resultado, han desarrollado mejores prácticas dentro de sus redacciones. Evidentemente, como se señaló, esta situación aún no es generalizable a todo el sistema mediático mexicano, el cual presenta tanto avances como estancamientos en su operación. Sin embargo, con respecto a las agresiones contra la prensa, el análisis se vuelve aún más complejo, porque la región donde trabajan los informantes no parece ser un factor determinante para la profesionalización. Esto es, los hallazgos no indican diferencias significativas de la práctica periodística relacionadas con la zona donde trabajan los entrevistados. Una explicación tentativa es que el riesgo al que se enfrentan los periodistas es constante y generalizado en todo el país, por lo que hay una sensación de indefensión y vulnerabilidad que permea entre los miembros del gremio, independientemente de si viven en la Ciudad de México o en un municipio alejado de la capital de cualquier estado. Evidentemente, hay entidades más peligrosas que otras, pero los ataques contra la prensa, especialmente desde 2017, se han incrementado en lugares que otrora eran considerados como santuarios (Article 19, 2019; CPJ, 2019). Y es que, como se mencionó, la impunidad y la corrupción generalizadas facilitan el incremento sostenido de los ataques. Por tanto, al menos en este caso, la tendencia a la profesionalización o autocensura es más resultado de decisiones individuales y organizacionales que del contexto regional.

\section{Referencias}

Article 19. (2019). ARTICLE 19 presenta el informe especial "Protocolo de la Impunidad en Delitos contra Periodistas". https://articulo19.org/ informeimpunidad/

Brambila, J. (2017). Forced silence: Determinants of journalists killings in Mexico's states. 2010-2015. Journal of Information Policy, 7, 297326. https://doi.org/10.5325/jinfopoli.7.2017.0297

Committee to Protect Journalists. (2019). 111 Journalists and Media Workers Killed in Mexico Between 1992 and 2019/Motive Confirmed or Unconfirmed. https://cpj.org/data/killed/?status=Killed\&motiv 
eConfirmed\%5B\%5D=Confirmed\&motiveUnconfirmed\%5B\%5 $\mathrm{D}=$ Unconfirmed\&type $\% 5 \mathrm{~B} \% 5 \mathrm{D}=$ Journalist\&type $\% 5 \mathrm{~B} \% 5 \mathrm{D}=\mathrm{Me}$ dia\%20Worker\&cc_fips\%5B\%5D=MX\&start_year=1992\&end year=2019\&group_by=year

Cottle, S., Sambrook, R. y Mosdell, N. (2016). Reporting dangerously: Journalist killings, intimidation and security. Palgrave McMillan.

De Albuquerque, A. (2019). Journalism and multiple modernities: The Folha de S. Paulo reform in Brazil. Journalism Studies, 20(11), 15461562. https://doi.org/10.1080/1461670X.2018.1528881

Del Palacio, C. (2018). Callar o morir en Veracruz: Violencia y medios de comunicación en el sexenio de Javier Duarte (2010-2016). Juan Pablos Editor.

Deuze, M. (2005). What is journalism? Professional identity and ideology of journalists reconsidered. Journalism, 6(4), 442-464. https:// doi.org/10.1177/1464884905056815

Eisenstadt, S. N. (2000). Multiple modernities. Daedalus, 129(1), 1-29.

Gans, H. (2004). Deciding what's news: A study of CBS Evening News, NBC Nightly News, Newsweek and Time. Northwestern University Press.

González-Macías, R. A. (2018). Silence or alignment: Organized crime and government as primary definers of news in Mexico. Observatorio $\left(\mathrm{OBS}^{*}\right)$ Journal, 12(4), 125-139. http://www.scielo.mec.pt/ scielo.php?script=sci_arttext\&pid=S1646-59542018000400008

González Macías, R. A. y Echeverría Victoria, M. (2018). A medio camino: El sistema mediático mexicano y su irregular proceso de modernización. Revista Mexicana de Opinión Pública, 13(24), 35-51. https://doi.org/10.22201/fcpys.24484911e.2018.24.60437 
González-Macías, R. A. y Reyna-García, V. H. (2019). “They don’t trust us; they don't care if we're attacked": Trust and risk perception in Mexican journalism. Communication y Society, 32(1), 147-160. https://dadun.unav.edu/handle/10171/57838

Hallin, D. C. y Mancini, P. (2004). Comparing media systems: Three models of media and politics. Cambridge University Press.

Holland, B. E. y Ríos, V. (2015). Informally governing information: How criminal rivalry leads to violence against press in Mexico. Journal of Conflict Resolution, 61(5), 1095-1119. https:// doi. org/10.1177/0022002715600756

Hughes, S. L. y Márquez Ramírez, M. (2017). Examining the practices that Mexican journalists employ to reduce risk in a context of violence. International Journal of Communication, 11, 499-521. http://192.203.177.185/bitstream/handle/ibero/1532/MRM Art_01.pdf? sequence $=1$ \&isAllowed $=y$

Hughes, S. L. y Márquez-Ramírez, M. (2018). Local-level authoritarianism, democratic normative aspirations, and antipress harassment: Predictors of threats to journalists in Mexico. The International Journal of Press/Politics, 23(4), 539-560. https://doi. org/10.1177/1940161218786041

Hughes, S. L., Mellado, C., Arroyave, J., Benitez, J. L., de Beer, A., Garcés, M., Lang, K. y Márquez-Ramírez, M. (2017). Expanding influences research to insecure democracies: How violence, public insecurity, economic inequality and uneven democratic performance shape journalists' perceived work environments. Journalism Studies, 18(5), 645-665. https://doi.org/10.1080/146167 0X.2016.1266278

Mancini, P. y Swanson, D. L. (1996). Politics, media, and modern democracy: Introduction. En P. Mancini y D. L. Swanson (eds.), 
Politics, media, and modern democracy: An international study of innovations in electoral campaigning and their consequences (pp. 1-26). Praeger Publisher.

Meijer, I. C. (2012). Valuable journalism: A search for quality from the vantage point of the user. Journalism, 14(6) 754-770. https://doi. org/10.1177/1464884912455899

Portes, A. (1973). Modernity and development: A critique. Comparative International Development, 8(3), 247-279. https://doi.org/10.1007/ BF02800432

Relly, J. E. y González de Bustamante, C. (2014). Silencing Mexico: A study of influences on journalists in the Northern states. The International Journal of Press/Politics, 19(1), 108-131. https://doi. org/10.1177/1940161213509285

Relly, J.E. y González de Bustamante, C. (2017). Global and domestic networks advancing prospects for institutional and social change: The collective action response to violence against journalists. Journalism y Communication Monographs, 19(2), 84-152. https://doi. org/10.1177/1522637917702618

Reyna García, V.H. (2014). Nuevos riesgos, viejos encuadres: La escenificación de la inseguridad pública en Sonora. El Colegio de Sonora.

Salazar Rebolledo, M. G. (2018). ¿Cuarto poder? Mercados, audiencias y contenidos en la prensa estatal mexicana. Politica y Gobierno, 25(1), 125-152.http://www.scielo.org.mx/scielo.php?script=sci_arttex t\&pid=S1665-20372018000100125

Shoemaker, P. y Reese, S. D. (1996). Mediating the message: Theories of influences on mass media content. Longman.

Sparks, C. (2011). Media and transition in Latin America. Westminster Papers in Communication and Culture, 8(2), 3-41. http://citeseerx. 
ist.psu.edu/viewdoc/download?doi=10.1.1.462.8796\&rep=rep $1 \&$ type $=$ pdf $\#$ page $=7$

Tuchman, G. (1972). Objectivity as a strategic ritual: An Examination of Newsmen's Notions of Objectivity. American Journal of Sociology, 77(4), 660-679. https://doi.org/10.1086/225193

Tuchman, G. (1978). Making news: A study in the construction of reality. Free Press.

Voltmer, K. (2012). How far can media systems travel? Applying Hallin and Mancini's comparative framework outside the Western world. En D. Hallin y P. Mancini (eds.), Comparing media systems beyond the Western world (pp. 224-245). Cambridge University Press.

Waisbord, S. (2006). In journalism we trust? Credibility and fragmented journalism in Latin America. En K. Voltmer (ed.), Mass media and political communication in new democracies (pp. 64-77). Routledge. 